\title{
The impact of low preoperative fat-free body mass on infections and length of stay after cardiac surgery: A prospective cohort study
}

\author{
Lenny M. W. van Venrooij, RD, MSc, ${ }^{\text {a,b }}$ Rien de $\operatorname{Vos}, \mathrm{PhD},{ }^{\mathrm{c}}$ Evelien Zijlstra, MSc, ${ }^{\mathrm{d}}$ \\ Mieke M. M. J. Borgmeijer-Hoelen, MD, ${ }^{\mathrm{b}}$ Paul A. M. van Leeuwen, MD, PhD, ${ }^{\mathrm{e}}$ and Bas A. J. M. de \\ Mol, MD, $\mathrm{PhD}^{\mathrm{b}}$
}

\begin{abstract}
Objective: Several studies have shown low fat-free mass index to be a stronger predictor for mortality than low body mass index. The main aim of this study was to assess the still unknown association between preoperative low fat-free mass index and adverse cardiac surgical outcomes.
\end{abstract}

\begin{abstract}
Methods: In a prospective observational study, fat-free mass index was determined by bioelectric impedance spectroscopy on hospital admission. Associations between low fat-free mass index and postoperative infections and mortality, as well as prolonged intensive care unit and hospital stays, were analyzed with logistic and Cox regression techniques.
\end{abstract}

\begin{abstract}
Results: Between February 2008 and December 2009, 325 adult patients admitted for elective heart surgery were included. Analyses showed that low fat-free mass index, present in $8.3 \%$ of patients, was independently associated with occurrence of infections after cardiac surgery (18.5\% vs $4.7 \%$; adjusted odds ratio, $6.9 ; 95 \%$ confidence interval, $1.8-27.7 ; P=.01)$. Low fat-free mass index also tended to be associated with higher risk of longer postoperative intensive care unit stay (adjusted hazard ratio, $0.7 ; 95 \%$ confidence interval, $0.4-1.1$; $P=.09$ ). When classifying patients as undernourished by traditional methods (body mass index $\leq 21.0$ $\mathrm{kg} / \mathrm{m}^{2}$ or $\geq 10 \%$ weight loss in preceding 6 months), half of patients with low fat-free mass index were misclassified as well nourished.
\end{abstract}

Conclusions: Low fat-free mass index is associated with increased occurrence of adverse outcomes after cardiac surgery. We advocate fat-free mass index as the leading parameter in classifying and treating undernourished cardiac surgical patients, which might improve recovery rates after cardiac surgery. (J Thorac Cardiovasc Surg 2011;142:1263-9)

Disease-related undernutrition is associated with higher postoperative risks of infectious and noninfectious complications, increased morbidity and mortality, and prolonged hospital stay; it can also lead to impaired wound healing. ${ }^{1,2}$ In The Netherlands, undernutrition is present in about $25 \%$ to $40 \%$ of hospital inpatients and in $10 \%$ to $20 \%$ of the preoperative outpatient population. ${ }^{3,4}$

In cardiac surgery, $10 \%$ to $25 \%$ of patients are undernourished before the operation. ${ }^{2,5}$ Cardiac surgical patients with a low preoperative body mass index (BMI) or unintended weight loss are at higher risk of postoperative

\footnotetext{
From the Departments of Dietetics, ${ }^{\text {a }}$ Cardiothoracic Surgery, ${ }^{\mathrm{b}}$ and Clinical Epidemiology and Biostatistics, ${ }^{\mathrm{c}}$ Academic Medical Center, University of Amsterdam, The Netherlands; the Institute of Health Sciences, ${ }^{\mathrm{d}}$ Faculty Earth and Life Sciences, VU University, Amsterdam, The Netherlands; and the Department of Surgery, ${ }^{\mathrm{e}} \mathrm{VU}$ University Medical Center Amsterdam, Amsterdam, The Netherlands.

Disclosures: Authors have nothing to disclose with regard to commercial support.

Received for publication Jan 3, 2011; revisions received June 27, 2011; accepted for publication July 19, 2011; available ahead of print Aug 22, 2011.

Address for reprints: Lenny M. W. van Venrooij, RD, MSc, Department of Cardiothoracic Surgery, Room G3-152, Academic Medical Center, P.O. Box 22700, 1100 DE Amsterdam, The Netherlands (E-mail: 1.m.vanvenrooij@amc.uva.nl). $0022-5223 / \$ 36.00$

Copyright (C) 2011 by The American Association for Thoracic Surgery doi:10.1016/j.jtcvs.2011.07.033
}

complications, infections, and mortality than are obese or even severely obese patients. ${ }^{2,5,6}$ Although BMI and weight loss help to identify the undernourished patients, most probably because BMI and weight loss are related to fat-free mass index (FFMI) ${ }^{7}$ they do not give exact information on body composition.

Body mass consists of metabolically active fat-free mass and inactive fat mass. In studies in a mixed hospital population and on major vascular surgery, a low FFMI has been shown to be associated with a longer hospital stay and an increased occurrence of the systemic inflammatory response syndrome, respectively. ${ }^{8,9}$ It has been demonstrated in patients with chronic obstructive pulmonary disease that a low FFMI is a stronger predictor for mortality than is a low BMI. ${ }^{10}$ Patients with a normal BMI may still have undernutrition because of a low fat-free mass. Thus if only BMI and weight loss are used to identify undernourished patients, some cardiac surgical patients with a low FFMI might be misclassified as well nourished. It is evident that these misclassifications could lead to inappropriate nutritional treatment and possible postoperative complications. Both overfeeding of the well-nourished patient and untreated undernutrition result in more complications after cardiac surgery. ${ }^{5,11}$ 


\section{Abbreviations and Acronyms \\ BMI = body mass index \\ CI = confidence interval \\ FFMI $=$ fat-free mass index \\ ICU = intensive care unit \\ $\mathrm{OR}=$ odds ratio}

In contrast to BMI and weight loss, the association between a low preoperative FFMI and the occurrence of postoperative infections has not yet been established, either in cardiac surgical patients or, to the best of our knowledge, in other surgical populations. The main aim of this study was therefore to assess the association between a low preoperative FFMI and the occurrence of infections and mortality as well as the durations of intensive care unit (ICU) and hospital stays after cardiac surgery. Secondarily, the additional value of measuring low FFMI in addition to the most commonly used parameters to identify undernourished patients—-low BMI and unintended weight loss—was assessed.

\section{MATERIALS AND METHODS \\ Subjects and Design}

Between February 2008 and December 2009, a prospective cohort study was conducted. Consecutive patients admitted to the cardiothoracic surgical ward at the Academic Medical Center for elective heart surgery (coronary artery bypass grafting, heart valve surgery, or both) with extracorporeal circulation were asked to participate. Those patients who were not willing or able to give written, informed consent, who had a pacemaker or a congenital heart abnormality, or who had undergone heart surgery within the preceding 3 months were excluded. This study was approved by the medical ethical committee of the Academic Medical Center, Amsterdam, The Netherlands.

\section{Body Composition}

On the day of admission to the cardiothoracic surgical ward, preoperative patient baseline measurements were taken. Data were collected on height, weight, and body composition while patients were barefoot and in underwear. Body height was measured to the nearest $0.5 \mathrm{~cm}$ with a stadiometer (Seca Deutschland, Hamburg, Germany). Current body weight was measured with an electronic beam scale with digital readout to the nearest $0.1 \mathrm{~kg}$ (Inventum Scala PW200; Inventum Group BV, Veenendaal, The Netherlands). Patients were asked for their weights at 1 and 6 months before admission. Body composition, (fat-free mass) was determined by bioelectric impedance spectroscopy (BIS; BodyScout; Fresenius Kabi AG, Bad Homburg, Germany). BIS measurements were done while the patients were lying supine with legs apart and arms abducted. BIS measurements were performed only once on a given patient. BIS measurements are highly reproducible and operator independent. ${ }^{12}$ Measurement was performed on the right side of the body with 4 electrodes (3M Red Dot; 3M Medica $\mathrm{GmbH}$, Borken, Germany); 2 electrodes were placed on the dorsum of the hand and 2 on the dorsum of the foot. A 5- to 1000-kHz electric current was briefly introduced into the body, and tissue conductivity was measured. Multiple frequencies enabled measurement of both intracellular fluid and extracellular fluid separately. Subsequently, fat-free mass was calculated. ${ }^{13}$ To adjust for differences in body height, FFMI was calculated by dividing fat-free mass in kilograms by squared height in square meters.

\section{Undernutrition}

Patients were classified as undernourished in terms of a low FFMI according to the cutoff points suggested by the research of Kyle and colleagues ${ }^{14}$ (FFMI $\leq 14.6 \mathrm{~kg} / \mathrm{m}^{2}$ in women and $\leq 16.7 \mathrm{~kg} / \mathrm{m}^{2}$ in men). This healthy population study $(\mathrm{n}=5635)$ shows FFMI values of 16.7 to 19.8 $\mathrm{kg} / \mathrm{m}^{2}$ for men and 14.6 to $16.8 \mathrm{~kg} / \mathrm{m}^{2}$ for women within normal BMI ranges $\left(18.5-25 \mathrm{~kg} / \mathrm{m}^{2}\right) .{ }^{14}$ Patients undergoing cardiac surgery were classified as undernourished according to BMI if BMI was not more than $21.0 \mathrm{~kg} / \mathrm{m}^{2}$ and according to unintended weight loss if weight loss had been at least $10 \%$ in the preceding 6 months. ${ }^{5}$

\section{Patient Characteristics}

Patient characteristics such as age, sex, operative procedure, operative risk, inflammatory activity, hypoalbuminemia, heart failure, and operative time were extracted from medical files and the standard electronic database of the cardiothoracic surgery department.

For statistical analysis, age was modeled as both a continuous variable and a dichotomized variable of at least 65 years versus less than 65 years. The operative procedure was dichotomized as isolated coronary artery bypass grafting versus isolated heart valve surgery or both. Operative risk was dichotomized as a EuroSCORE value of at least 6 (high operation risk) versus a score less than 6 (low risk). Inflammatory activity was dichotomized as C-reactive protein of at least $5 \mathrm{mg} / \mathrm{L}$ versus less than 5 $\mathrm{mg} / \mathrm{L}$, hypoalbuminemia as less than or equal to $39 \mathrm{~g} / \mathrm{L}$ versus more than $39 \mathrm{~g} / \mathrm{L}$, and heart failure as prohormone brain natriuretic peptide of at least $600 \mathrm{ng} / \mathrm{L}$ versus less than $600 \mathrm{ng} / \mathrm{L}$. Operative time was divided into cardiopulmonary bypass time and aortic crossclamp time and dichotomized as at least 130 minutes versus less than 130 minutes for cardiopulmonary bypass time and at least 90 minutes versus less than 90 minutes for aortic crossclamp time.

\section{Postoperative Adverse Outcomes}

Data about postoperative complications were also collected from medical files and from the standard electronic database of the cardiothoracic surgery department at the Academic Medical Center. The postoperative adverse outcomes were the occurrence of postoperative infections and death as well as durations of ICU and hospital stays, all during the period of hospitalization for surgery. Additionally, these outcome data were completed with data covering the complete hospitalization period directly after transfer from the hospital where surgery was carried out back to the referring hospital. Postoperative infection was defined as the composite of septicemia, respiratory tract infection, mediastinitis, deep sternal wound infection, and leg wound infection. These infectious complications were in accordance with the definitions of The Society of Thoracic Surgeons (version 2.61, 2007; http://www.sts.org). To assess the associations between a low FFMI and ICU and hospital stays, these outcome data were modeled as continuous variables, with ICU stay in hours and hospital stay in days. Postoperative death was defined as death during hospital admission.

\section{Statistical Analyses}

To estimate the effect size of the association between a low preoperative FFMI and the occurrence of postoperative infections, first univariate logistic regression analysis was carried out. The odds ratio (OR) and $95 \%$ confidence interval $(95 \% \mathrm{CI})$ were calculated, with postoperative adverse outcome as a dependent variable and low FFMI as an independent variable. Second, if a $P$ value of .10 or less than .10 for low FFMI in the univariate logistic regression analysis was present, multivariate regression analysis adjusting for sex, age, operative procedure, operative risk, heart failure, inflammatory activity, hypoalbuminemia, and operative time was performed. A similar procedure was conducted to assess the association between a low preoperative FFMI and mortality. To include time-related information concerning durations of ICU and hospital stays, these 
dependent outcome variables were analyzed with univariate and multivariate Cox regression analyses adjusting for sex, age, operative procedure, operative risk, heart failure, inflammatory activity, hypoalbuminemia, and operative time. Discharge at any point during the admission period was coded as an event, and death or unavailability for follow-up was coded as censored data. The proportional hazard assumptions of Cox regression analysis were met. Hazard ratios and their $95 \%$ CIs were calculated. Finally, to determine the independent value of a low FFMI in addition to the traditional way of identifying the undernourished (BMI $\leq 21.0 \mathrm{~kg} / \mathrm{m}^{2}$ or $\geq 10 \%$ weight loss in the preceding 6 months), we also entered low BMI and weight loss into the previously mentioned multivariate logistic and Cox regression models. To demonstrate the additional value of classifying patients as being undernourished with a low FFMI instead of low BMI or recent weight loss, crosstabs were presented. All statistical analyses were done with SPSS statistical software for Windows (version 16.0; IBM Corporation, Armonk, NY).

\section{RESULTS \\ Subjects}

Between February 2008 and December 2009, a total of 770 patients undergoing cardiac surgery were eligible for inclusion. Between June and October 2008, inclusion was interrupted for logistic reasons $(n=162)$. In addition, 174 patients could not be asked to join because of logistic interference with the usual preoperative medical assessment protocol. Two patients were excluded because they had no command of the Dutch language, and in 3 cases no body weight and height measurements could taken, because the patients were confined to bed. In 33 cases, no BIS (FFMI) measurements could be performed because of the presence of a pacemaker.

A total of 396 patients were asked to participate. Of these patients, $17.9 \%(\mathrm{n}=71)$ refused to participate. These patients had a higher operative risk and were older than those patients who gave informed consent $(P<.005$ and $P=.01$, respectively). The remaining data from 325 patients were analyzed. Their preoperative baseline characteristics are summarized in Table 1.

\section{Preoperative Undernutrition and Low FFMI}

Preoperatively, $13.0 \%$ of cardiac surgical patients $(\mathrm{n}=42)$ were undernourished: $8.3 \%(\mathrm{n}=27)$ had a low FFMI, $4.0 \%$ had a BMI of $21.0 \mathrm{~kg} / \mathrm{m}^{2}$ or less, and $5.3 \%$ had at least $10 \%$ weight loss in the preceding 6 months. A low FFMI was largely present in women $(16.7 \%$ vs $5.1 \%)$, elderly patients $(11.3 \%$ vs $4.3 \%)$, patients at high operative risk patients $(15.0 \%$ vs $4.0 \%)$, patients undergoing heart valve surgery $(11.5 \%$ vs $5.4 \%)$, and patients with severe heart failure $(15.0 \%$ vs $5.0 \% ; P \leq .05)$. No associations were found with inflammatory activity or hypoalbuminemia.

\section{Low FFMI and Postoperative Adverse Outcomes}

Postoperative infections occurred in 19 patients $(5.8 \%)$, and eight patients $(2.5 \%)$ died during admission to the operating hospital (Table 2). Most of the infections
TABLE 1. Preoperative baseline characteristics of the study cohort $(\mathbf{n}=\mathbf{3 2 5})$

\begin{tabular}{|c|c|}
\hline Female sex (no., \%) & $90(27.7)$ \\
\hline \multicolumn{2}{|l|}{ Age (y) } \\
\hline Mean $\pm \mathrm{SD}$ & $66.2 \pm 9.8$ \\
\hline$\geq 65$ (no.) & $186(57.2)$ \\
\hline \multicolumn{2}{|l|}{$\operatorname{BMI}\left(\mathrm{kg} / \mathrm{m}^{2}\right)$} \\
\hline Mean \pm SD & $27.2 \pm 4.1$ \\
\hline$\geq 30.0($ no., \%) & $63(19.4)$ \\
\hline Left ventricular ejection fraction $<30 \%$ (no., $\%$ ) & $8(2.5)$ \\
\hline Diabetes mellitus (no., \%) & $70(21.7)$ \\
\hline Chronic obstructive pulmonary disease (no., \%) & $24(7.6)$ \\
\hline Smoking (no., \%) & $62(19.1)$ \\
\hline Pulmonary hypertension (no., \%) & $7(2.2)$ \\
\hline Recent myocardial infarction (no., \%) & $28(8.6)$ \\
\hline Extracardiac arteriopathy (no., \%) & $24(7.4)$ \\
\hline \multicolumn{2}{|l|}{ EuroSCORE } \\
\hline 0-2 (low risk; no., \%) & 89 (27.4) \\
\hline 3-5 (medium risk; no., \%) & $109(33.5)$ \\
\hline$\geq 6$ (high risk; no., $\%$ ) & $127(39.1)$ \\
\hline \multicolumn{2}{|l|}{ Laboratory values } \\
\hline Serum creatinine $\geq 200 \mu \mathrm{mol} / \mathrm{L}$ (no., $\%$ ) & $2(0.6)$ \\
\hline $\begin{array}{l}\text { Prohormone brain natriuretic peptide (marker for } \\
\text { heart failure) } \geq 600 \mathrm{ng} / \mathrm{L} \text { (no., } \% \text { ) }\end{array}$ & $107(32.9)^{*}$ \\
\hline C-reactive protein $\geq 5 \mathrm{mg} / \mathrm{L}$ (no., $\%$ ) & $53(16.3)$ \\
\hline Albumin $\leq 39 \mathrm{~g} / \mathrm{L}$ (no., \%) & $12(3.7) \dagger$ \\
\hline \multicolumn{2}{|l|}{ Operative procedure } \\
\hline Coronary artery bypass grafting (no., \%) & $168(51.7)$ \\
\hline Heart valve surgery (no., \%) & $105(32.3)$ \\
\hline Both (no., \%) & $52(16.0)$ \\
\hline \multicolumn{2}{|l|}{ Duration of extracorporeal circulation } \\
\hline Cardiopulmonary bypass time $\geq 130 \mathrm{~min}$ (no., \%) & $106(32.6)$ \\
\hline Aortic crossclamp time $\geq 95 \min ($ no., $\%$ ) & $96(29.5)$ \\
\hline
\end{tabular}

(17/19) were of the respiratory tract. The cumulative incidence of postoperative infection or death was $7.7 \%$ $(\mathrm{n}=25)$.

Univariate analysis showed that a low preoperative FFMI was associated with the occurrence of postoperative infections $(18.5 \%$ vs $4.7 \%$; OR, $4.5 ; 95 \%$ CI, $1.5-13.8$; $P=.01$; Tables 2 and 3). After multivariate logistic regression analysis adjusting for operative risk, heart failure, and other prognostic factors, the association between a low preoperative FFMI and the occurrence of infections after cardiac surgery persisted (adjusted OR, $6.9 ; 95 \%$ CI, $1.8-27.2 ; P=.01$; Table 3 ). Also, during the entire hospitalization period, logistic regression analyses showed that a low preoperative FFMI was independently associated with an increased occurrence of infections $(31.8 \%$ vs $10.6 \%$; adjusted $\mathrm{OR}, 3.8 ; 95 \% \mathrm{CI}, 1.3-11.6 ; P=.02$; Tables 2 and 3). With respect to mortality, univariate analysis showed that a preoperatively low FFMI was associated with the occurrence of postoperative death during hospital admission but only for the entire hospitalization period $(13.6 \%$ vs $2.3 \%$; OR, $6.6 ; 95 \%$ 
TABLE 2. The occurrence of postoperative infections and death after cardiac surgery among patients with and without a low preoperative fat-free mass index

\begin{tabular}{|c|c|c|c|c|}
\hline & All (no., \%) & Low FFMI $(\%)$ & No low FFMI $(\%)$ & $P$ value \\
\hline \multicolumn{5}{|c|}{ Postoperative outcome during initial hospital admission in which surgery was carried out } \\
\hline Mortality* & $8(2.5)$ & 7.4 & 2.0 & .14 \\
\hline Infection & $19(5.8)$ & 18.5 & 4.7 & .01 \\
\hline Septicemia & $1(0.3)$ & 0 & 0.3 & - \\
\hline Respiratory tract infection & $17(5.2)$ & 18.5 & 4.0 & .01 \\
\hline Mediastinitis & $0(0)$ & - & - & - \\
\hline Deep sternal wound infection & $0(0)$ & - & - & - \\
\hline Leg wound infection & $1(0.3)$ & 0 & 0.3 & - \\
\hline \multicolumn{5}{|c|}{$\begin{array}{l}\text { Postoperative outcome data completed with data covering complete hospitalization period directly after transfer from hospital where surgery was carried out } \\
\text { back to referring hospital }\end{array}$} \\
\hline Mortality $\dagger$ & $9(3.2)$ & 13.6 & 2.3 & .03 \\
\hline Infection & $35(12.3)$ & 31.8 & 10.6 & .01 \\
\hline Septicemia & $1(0.4)$ & 0 & 0.4 & - \\
\hline Respiratory tract infection & $24(8.4)$ & 27.3 & 6.8 & .01 \\
\hline Mediastinitis & $4(1.4)$ & 4.5 & 1.1 & .28 \\
\hline Deep sternal wound infection & $1(0.4)$ & 0 & 0.4 & - \\
\hline Leg wound infection & $5(1.8)$ & 0 & 1.9 & - \\
\hline
\end{tabular}

Low fat-free mass index considered to be $\leq 14.6 \mathrm{~kg} / \mathrm{m}^{2}$ in women and $\leq 16.7 \mathrm{~kg} / \mathrm{m}^{2}$ in men $(8.3 \%, \mathrm{n}=27)$. FFMI, Fat-free mass index. $*$ All 8 patients who died in initial hospitalization died within 30 days from surgery (range, $0-25$ days). $\dagger$ Additional patient died 43 days after surgery.

CI, 1.5-28.8; Tables 2 and 3). Mortality was not further analyzed because of its low prevalence $(3.2 \%, \mathrm{n}=9)$.

With respect to durations of hospital and ICU stays, a low FFMI tended to be associated with a higher risk of a longer postoperative stay in the ICU (adjusted hazard ratio, 0.7; 95\% CI, 0.4-1.1; $P=.09$; Table 3 and Figure 1). No patients were readmitted to the ICU during the hospitalization period after transfer back to the referring hospital. Univariate analysis showed associations between low FFMI and hospital stay during admission at the operating hospital and during the entire hospital admission; however, these associations could be completely explained by adjusting for the factors of sex, age, operative procedure, operative risk, heart failure, inflammatory activity, hypoalbuminemia, and operative time (Table 3 ).
In 40 cases $(12.3 \%)$, data covering the entire hospitalization period were missing. Sex, age, operative procedure, risk, and FFMI did not differ between patients included and those unavailable for follow up.

\section{Low FFMI in Relation to the Traditional Means of Identifying Undernutrition}

After multivariate modeling including undernutrition defined by both low BMI and unintended weight loss and the other prognostic factors, a preoperatively low FFMI was still independently associated with the occurrence of postoperative infections during hospital stay at the operating hospital and during total hospital stay including admission to the referring hospital (adjusted OR, 7.5; 95\% CI, 1.6$34.8 ; P=.01 ;$ and adjusted OR, 5.0; 95\% CI, 1.4-18.1;

TABLE 3. Univariate and multivariate regression analyses of low preoperative fat-free mass index and the occurrence of infections, prolonged intensive care unit stay, and prolonged hospital stay after cardiac surgery

\begin{tabular}{|c|c|c|c|c|c|c|}
\hline & OR/HR & $95 \% \mathrm{CI}$ & $P$ value & Adjusted OR/HR* & $95 \% \mathrm{CI}$ & $P$ value \\
\hline \multicolumn{7}{|l|}{ Logistic regression analysis (OR) } \\
\hline Infection $[\mathrm{AMC}](5.8 \%, \mathrm{n}=19)$ & 4.52 & $1.49-13.78$ & .01 & 6.89 & $1.75-27.17$ & .01 \\
\hline Infection [OVERALL] $(12.3 \%, \mathrm{n}=35)$ & 3.91 & $1.46-10.51$ & .01 & 3.83 & $1.27-11.6$ & .02 \\
\hline Mortality [OVERALL] $(3.2 \%, \mathrm{n}=9)$ & 6.64 & $1.53-28.77$ & .01 & - & - & - \\
\hline \multicolumn{7}{|l|}{ Cox regression analysis (HR) } \\
\hline $\begin{array}{l}\text { Discharge from ICU [AMC] (median ICU stay, } 30 \mathrm{~h} \text {; } \\
\text { 10th-90th percentile, } 21-112 \mathrm{~h} \text { ) }\end{array}$ & 0.56 & $0.36-0.86$ & .01 & 0.67 & $0.42-1.07$ & .09 \\
\hline $\begin{array}{l}\text { Discharge from hospital [AMC] (median hospital } \\
\text { stay, } 5 \mathrm{~d} ; 10 \text { th-90th percentile, } 4-12 \mathrm{~d} \text { ) }\end{array}$ & 0.65 & $0.43-0.99$ & .05 & 0.81 & $0.52-1.27$ & .36 \\
\hline $\begin{array}{l}\text { Discharge from hospital [OVERALL] (median } \\
\text { hospital stay, } 10 \mathrm{~d} \text {; 10th-90th percentile, 7-18 d) }\end{array}$ & 0.60 & $0.37-0.97$ & .04 & 0.88 & $0.53-1.47$ & .36 \\
\hline
\end{tabular}

Low fat-free mass index considered to be $\leq 14.6 \mathrm{~kg} / \mathrm{m}^{2}$ in women, $\leq 16.7 \mathrm{~kg} / \mathrm{m}^{2}$ in men $(8.3 \%, \mathrm{n}=27)$. OR, Odds ratio; $H R$, hazard ratio; $C I$, confidence interval; $A M C$, Academic Medical Center, postoperative outcome during hospital admission in which surgery was carried out; OVERALL, postoperative outcome data completed with data covering complete hospitalization period directly following transfer from hospital where surgery was carried out back to referring hospital; ICU, intensive care unit. $*$ Multivariate models adjusted for sex, age, operative procedure, operative risk, heart failure, inflammatory activity, hypoalbuminemia and operative time. 


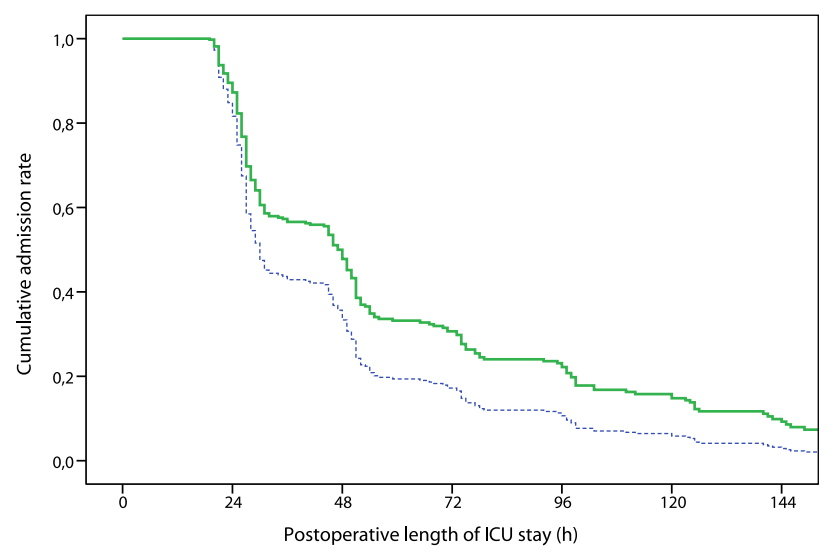

FIGURE 1. Postoperative admission rates to the intensive care unit (ICU) among patients undergoing cardiac surgery with low fat-free mass index (green line) and without low fat-free mass index (blue dotted line). Low fat-free mass index was determined according to the definition of Kyle and colleagues. ${ }^{14}$

$P=.02$, respectively). Also, independent of low BMI and unintended weight loss, a low preoperative FFMI still had a tendency to be associated with a higher risk of a longer postoperative stay in the ICU (adjusted hazard ratio, 0.6; $95 \%$ CI, $0.4-1.0 ; P=.06$ ).

When classifying undernutrition according to low FFMI instead of low BMI and weight loss, it was demonstrated that half of the patients with a low FFMI (13/27) were misclassified as well nourished if low BMI and weight loss alone were used to identify undernutrition (Table 4). The other way around, 15 of 29 patients classified as undernourished according to low BMI and weight loss did not have low FFMI. In 3 cases, no data were available on body weight 6 months before the hospital admission. None of these patients had a low FFMI.

\section{DISCUSSION}

To the best of our knowledge, this study is the first to demonstrate that undernutrition in terms of a low preoperative FFMI is, independent of other prognostic risk factors, associated with a higher risk of development of an infection after cardiac surgery. Patients with a low FFMI also tended to have a higher risk of a longer postoperative stay in the ICU. A low FFMI was seen in $8.3 \%$ of patients undergoing cardiac surgery. If low BMI and weight loss only were used to identify cardiac surgical patients who were undernourished preoperatively, half of patients with a low FFMI were misclassified as well nourished.

The association that we found between a low FFMI and the occurrence of adverse outcome in general is consistent with studies in other areas of medicine. ${ }^{8-10}$ A plausible explanation is that a low FFMI represents insufficient nutritional reserve. This implies decreased lean body mass, in other words, less metabolically active body cell
TABLE 4. Agreement between low fat-free mass index and low body mass index and weight loss to identify undernourished patients

\begin{tabular}{lccr}
\hline & \multicolumn{2}{c}{ Undernourished by current guidelines } & \\
\cline { 2 - 3 } Low FFMI & Yes & No & Total \\
\hline Yes & 14 & 13 & 27 \\
No & 15 & 280 & 295 \\
Total & 29 & 293 & 322 \\
\hline
\end{tabular}

Current guidelines for undernourishment considered to be body mass index $\leq 21.0$ $\mathrm{kg} / \mathrm{m}^{2}$ or weight loss $\geq 10 \%$ in 6 months. Low fat-free mass index considered to be $\leq 14.6 \mathrm{~kg} / \mathrm{m}^{2}$ in women and $\leq 16.7 \mathrm{~kg} / \mathrm{m}^{2}$ in men $(8.3 \%, \mathrm{n}=27) . F F M I$, Fat-free mass index.

mass. Less metabolically active cell mass results in a longer convalescence period after surgery, because patients do not have an adequate response to operative stress and are unable to handle complications well. ${ }^{15,16} \mathrm{~A}$ low FFMI could be expected to be associated with all kinds of infections. ${ }^{2,9}$ The fact that in this study a low FFMI was associated with the occurrence of respiratory tract infections and not wound infections can be explained by the low incidence of wound infections and, more importantly, by the fact that obesity, which is less often accompanied by a low FFMI, is a well-established significant risk factor for the occurrence of wound infections after cardiac surgery. ${ }^{17}$ Also, after adjustment for chronic obstructive pulmonary disease and smoking, which are possible confounders specifically for the association with respiratory tract infections, the association between a low FFMI and infections persisted. Future intervention studies assessing FFMI and outcome must further probe the degree of causality between low FFMI and postoperative infections.

In addition to other studies, our study demonstrated that FFMI provides information to assist in making a prognosis beyond that provided by BMI and weight loss before surgery. ${ }^{7,8,10}$ Most likely, fat mass reduces the sensitivity of low BMI and weight loss in detecting nutritional depletion. ${ }^{7}$ In cardiac surgical patients in particular, who are often overweight or obese, the sensitivity of BMI in detecting undernutrition is expected to be inadequate. In line with this, our study results showed that half of our cardiac surgical patients with a low FFMI were misclassified as well nourished if only classified by low BMI and weight loss. In clinical practice, this implies that before cardiac surgery half of the patients with insufficient nutritional reserve are currently not receiving the nutritional intervention necessary to optimize their postoperative recovery. It was also observed that half of patients classified as undernourished on the basis of low BMI and weight loss alone did not have a low FFMI. This is not a consequence of inaccurate BIS measurements. Rather, it is because FFMI at a given time does not account for relevant losses of fat-free mass in the preceding months. Except for 1 patient, all patients who were classified as undernourished but did not have 
low FFMI had lost $10 \%$ or more body weight in the preceding 6 months. For effective identification and thereby treatment of preoperatively undernourished cardiac surgical patients, we therefore advocate the measurement of FFMI in addition to the traditional detection of undernutrition on the basis of recent weight loss and BMI.

It could be hypothesized that higher cutoff points for a low FFMI in cardiac surgery patients are needed, because overweight and obese individuals may have an apparently normal absolute quantity of fat-free mass, yet this fat-free mass might still be inadequate for their size. ${ }^{18}$ We therefore also analyzed the lower 5th, 15th, and 25th percentiles of FFMI derived from this cardiac surgical population sample. It was shown that the lower 15th percentile, FFMI less than or equal to $14.4 \mathrm{~kg} / \mathrm{m}^{2}$ in women $(\mathrm{n}=13)$ and less than or equal to $17.8 \mathrm{~kg} / \mathrm{m}^{2}$ in men $(\mathrm{n}=34)$, was independently associated with a higher risk of a longer postoperative stay in the ICU (adjusted hazard ratio, 0.7; 95\% CI, 0.5-1.0; $P=.03)$. Continued research to validate cardiac surgeryspecific cutoff points for a low FFMI is recommended.

The finding that undernourished cardiac surgical patients only very exceptionally look thin or correspond with general definitions of undernutrition underlines the absolute necessity of measuring body composition in cardiac surgical patients. To reduce the resulting extra workload and costs, it would be of value to develop a quick and easy cardiac surgery-specific nutritional screening tool that would easily identify those patients at higher risk of having a low FFMI. This study observed that a low FFMI was largely present in women, elderly patients, patients at high operative risk, patients undergoing heart valve surgery, and patients with severe heart failure.

Our study has some limitations. The total number of events was relatively low, and it cannot be excluded that results are context specific. We therefore invite other researchers to reproduce our study and test our preliminary findings. Furthermore, although it is reasonable to assume that BIS accurately measured fat-free mass, it should be realized that BIS is sensitive to hydration abnormalities. ${ }^{13}$ Analyzing our data, it was observed that decompensatio cordis was present in $8 \%$. This may have resulted in some bias and overestimation of the metabolically active part of fat-free mass and thus in underestimation of undernutrition.

Because about $10 \%$ to $25 \%$ of patients undergoing cardiac surgery either are or become undernourished, resulting in a higher risk of postoperative complications, prolonged admissions, and less quality of life, ${ }^{5,19}$ care should be taken that their preoperative and postoperative nutritional statuses are the best possible. Supplementation of essential amino acids increases lean body mass, muscle strength, and physical condition by stimulating muscle protein synthesis and improves postoperative outcome. ${ }^{20,21}$ In general, 10 to 14 days of preoperative nutritional support improves postoperative outcome in the undernourished patient. ${ }^{22}$ Because the majority of patients undergoing elective cardiac surgery have to wait for at least 14 days, nutritional treatment to improve insufficient preoperative nutritional reserve could easily be started in the period before the operation. Moreover, nutritional support to increase muscle protein synthesis seems most effective in combination with physical exercise. ${ }^{23}$ In the setting of cardiac rehabilitation and in elderly patients, early resistance training has been shown to be safe and of additional value to aerobic regimens to increase muscle strength and mass, clinical status, exercise capacity, and quality of life. ${ }^{24,25}$ We therefore suggest studying the effect of a program that focuses on resistance muscle training combined with nutritional interventions to overcome the cardiac surgery-induced catabolic response. This program should be started preoperatively, restarted in the early postoperative phase, and continued in the long term. For effective identification and thereby treatment of preoperatively undernourished cardiac surgical patients, we advocate, especially for highrisk patients, the measurement of FFMI in addition to the traditional method of assessing nutritional status with BMI and recent weight loss only, although whether BIS or dualenergy $\mathrm{x}$-ray absorptiometry is the instrument of choice for measurement of FFMI is still the subject of debate. ${ }^{13}$ As a result of implementing FFMI measurement, recovery rates after cardiac surgery might be improved.

We thank the nursing team and the secretarial staff of the department of Cardiothoracic Surgery at the Academic Medical Center, University of Amsterdam, and in particular Mrs Ella Oosterhof, for their assistance in instructing the participants. We also thank Cees Haaring, Trial Office, Department of Radiology, University Medical Center Utrecht, for his technical assistance in organizing and linking the preoperative and postoperative databases. Further, we thank Liesbeth, Amanda, Sonja, Tessa, Danja, Denise, Wendy, and Liska, all students at the time of the project, for their assistance in collecting data. We also thank Daphne Lees, editor, for her critical reading of this article. Last, but not least, we thank all patients willing to participate despite the stressful events in their lives. L.M.W.V. was responsible for the protocol, selection, data collection, analysis, and writing of the manuscript. R.V. participated in the methodologic development of the study protocol and coauthored the manuscript. E.Z. participated in the selection, data collection, and analysis and coauthored the manuscript. M.M.M.J.B. participated in the selection, data collection, and development of the protocol. P.A.M.L. participated in the development of the protocol and reviewed the manuscript. B.A.J.M.M. participated in the development of the protocol and reviewed the manuscript.

\footnotetext{
References

1. Correia MI, Waitzberg DL. The impact of malnutrition on morbidity, mortality, length of stay and costs evaluated through a multivariate model analysis. Clin Nutr. 2003;22:235-9.

2. Engelman DT, Adams DH, Byrne JG, Aranki SF, Collins JJ, Couper GS, et al. Impact of body mass index and albumin on morbidity and mortality after cardiac surgery. J Thorac Cardiovasc Surg. 1999;118:866-73.
} 
3. Meijers JM, Schols JM, Van Bokhorst-de van der Schueren MA, Dassen T, Janssen MA, Halfens RJ. Malnutrition prevalence in The Netherlands: results of the annual Dutch national prevalence measurement of care problems. $\mathrm{Br} J$ Nutr. 2009;101:417-23.

4. Leistra E, Neelemaat F, Evers AM, van Zandvoort MH, Weijs PJ, van Bokhorstde van der Schueren MA, et al. Prevalence of undernutrition in Dutch hospital outpatients. Eur J Intern Med. 2009;20:509-13.

5. Van Venrooij LM, De Vos R, Borgmeijer-Hoelen AM, Haaring C, de Mol BA. Preoperative unintended weight loss and low body mass index in relation to complications and length of stay after cardiac surgery. Am J Clin Nutr. 2008;87: 1656-61.

6. Thourani VH, Keeling WB, Kilgo PD, Puskas JD, Lattouf OM, Chen EP, et al. The impact of body mass index on morbidity and short- and long-term mortality in cardiac valvular surgery. J Thorac Cardiovasc Surg. Epub 2011 Mar 28.

7. Kyle UG, Pirlich M, Lochs H, Schuetz T, Pichard C. Increased length of stay in underweight and overweight patients at hospital admission: a controlled population study. Clin Nutr. 2005;24:133-42.

8. Pichard C, Kyle UG, Morabia A, Perrier A, Vermeulen B, Unger P. Nutritional assessment: lean body mass depletion at hospital admission is associated with an increased length of stay. Am J Clin Nutr. 2004;79:613-8.

9. Hassen TA, Pearson S, Cowled PA, Fitridge RA. Preoperative nutritional status predicts the severity of the systemic inflammatory response syndrome (SIRS) following major vascular surgery. Eur J Vasc Endovasc Surg. 2007;33:696-702.

10. Schols AM, Broekhuizen R, Weling-Scheepers CA, Wouters EF. Body composition and mortality in chronic obstructive pulmonary disease. Am J Clin Nutr. 2005;82:53-9.

11. Van Venrooij LM, Van Leeuwen PA, De Vos R, Borgmeijer-Hoelen AM, de Mol BA. Preoperative protein and energy intake and postoperative complications in well-nourished, non-hospitalized elderly cardiac surgery patients. Clin Nutr. 2009;28:117-21.

12. Ellis KJ. Human body composition: in vivo methods. Physiol Rev. 2000;80: 649-80.

13. Van Venrooij LM, Verberne HJ, De Vos R, Borgmeijer-Hoelen AM, Van Leeuwen PA, de Mol BA. Preoperative and postoperative agreement in fat free body mass (FFM) between the bioelectrical impedance spectroscopy (BIS) method and the dual energy X-ray absorptiometry (DXA) in patients undergoing cardiac surgery. Clin Nutr. 2010;29:789-94.
14. Kyle UG, Schutz Y, Dupertuis YM, Pichard C. Body composition interpretation Contributions of the fat free mass index and the body fat mass index. Nutrition. 2003; 19:597-604.

15. Soeters PB, Schols AM. Advances in understanding and assessing malnutrition Curr Opin Clin Nutr Metab Care. 2009;12:487-94.

16. Hill GL, Douglas RG, Schroeder D. Metabolic basis for the management of patients undergoing major surgery. World J Surg. 1993;17:146-53.

17. Bitkover CY, Gårdlund B. Mediastinitis after cardiovascular operations: a casecontrol study of risk factors. Ann Thorac Surg. 1998;65:36-40.

18. Zamboni M, Mazzali G, Fantin F, Rossi A, Di Francesco V. Sarcopenic obesity: a new category of obesity in the elderly. Nutr Metab Cardiovasc Dis. 2008;18 388-95.

19. Van Venrooij LM, Verberne HJ, De Vos R, Borgmeijer-Hoelen MM, Van Leeuwen PA, de Mol BA. Postoperative loss of skeletal muscle mass, complications and quality of life in patients undergoing cardiac surgery. Nutrition. Epub 2011 May 27.

20. Borsheim E, Bui QT, Tissier S, Kobayashi H, Ferrando AA, Wolfe RR. Effect of amino acid supplementation on muscle mass, strength and physical function in elderly. Clin Nutr. 2008;27:189-95.

21. Tepaske R, Velthuis H, Oudemans-van Straaten HM, Heisterkamp SH, van Deventer SJ, Ince C, et al. Effect of preoperative oral immune-enhancing nutritional supplement on patients at high risk of infection after cardiac surgery: a randomised placebo-controlled trial. Lancet. 2001;358:696-701.

22. Weimann A, Braga M, Harsanyi L, Laviano A, Ljungqvist O, Soeters P. ESPEN Guidelines on Enteral Nutrition: surgery including organ transplantation. Clin Nutr. 2006;25:224-44.

23. Biolo G, Ciocchi B, Lebenstedt M, Barazzoni R, Zanetti M, Platen P, et al. Shortterm bed rest impairs amino acid-induced protein anabolism in humans. $J$ Physiol. 2004;558(Pt 2):381-8.

24. Jankowska EA, Wegrzynowska K, Superlak M, Nowakowska K, Lazorczyk M Biel B, et al. The 12-week progressive quadriceps resistance training improves muscle strength, exercise capacity and quality of life in patients with stable chronic heart failure. Int J Cardiol. 2008;130:36-43.

25. Suetta C, Magnusson SP, Rosted A, Aagaard P, Jakobsen AK, Larsen LH, et al Resistance training in the early postoperative phase reduces hospitalization and leads to muscle hypertrophy in elderly hip surgery patients-a controlled, randomized study. J Am Geriatr Soc. 2004;52:2016-22. 